\title{
Two Female Nonsmoker Buerger's Disease Cases With Anticardiolipin Autoantibodies and a Poor Prognosis
}

\author{
Gulumser HePer, ${ }^{1} \mathrm{MD}$, Sedat Kose, ${ }^{2} \mathrm{MD}$, Ozkan AKKoc, ${ }^{1} \mathrm{MD}$, \\ Basri AMASYALI, ${ }^{2} \mathrm{MD}$, and Ayhan KILIC, ${ }^{2} \mathrm{MD}$
}

\begin{abstract}
SUMMARY
We present two female nonsmoker Buerger's disease cases with anticardiolipin autoantibodies and a poor prognosis. One was a 64-year-old female who has had multiple lower and upper extremity amputations, while the other was a 32-year-old female with extremity and visceral artery involvement. Since both were positive for anticardiolipin antibodies, we speculate that Buerger's disease is an autoimmune disorder. (Int Heart J 2005; 46: 563-569)
\end{abstract}

Key words: Buerger's disease, Anticardiolipin autoantibodies, Thromboangiitis obliterans

BUERGER'S disease is an inflammatory thrombotic nonatherosclerotic vascular disease that affects arterial and venous vessels of medium and small caliber and the nerves within the neurovascular bundle. This vasculitis affects mainly the lower limbs and then the upper limbs, but has also been described in the visceral vessels. It principally strikes young men who are heavy smokers. ${ }^{1,2)}$ It rarely affects women. Sasai, et al showed that nonsmokers at the first consultation were more common among women than among men. They also showed that clinical presentations in women with thromboangiitis obliterans (TAO) were almost equal to those in men. ${ }^{3)}$

The etiopathogenesis is still obscure, but smoking appears to be tightly linked. ${ }^{4)}$ We think there are possible immunoallergic or autoimmune reactions with glycoprotein antigens in tobacco.

The clinical criteria for diagnosis of TAO are a history of cigarette smoking, onset before age 50 years, infrapopliteal artery occlusive lesions, and the absence of atherosclerotic risk factors other than smoking. Arteriographic findings include tapering or abrupt occlusion of peripheral arterial vessels, a corkscrew or

From the ${ }^{1}$ Department of Cardiology, Social Security Hospital, and ${ }^{2}$ Department of Cardiology, Gulhane Military Medical Academy, Ankara, Turkey.

Address for correspondence: Gulumser Heper, MD, Onur Sokak 44/6 Maltepe, 06570, Ankara, Turkey.

Received for publication October 18, 2004.

Revised and accepted January 272005. 
rootlike appearance of collateral vessels, and a corrugated appearance of those leg arteries free of occlusive lesions.

\section{CASE 1}

A 64-year-old woman was observed for the first time for nonhealing right third finger pain and ulceration. Her past history was pertinent for complaints of Raynaud's syndrome and finger ulcers that did not heal for a prolonged period. She had no history of miscarriage or abortions. She had undergone three amputations. The first amputation was performed for a nonhealing great toe ulcer at the age of 34 years. The second amputation was performed just below her right knee for a foot ulcer and dry gangrene one year after her first amputation. The third amputation was performed on her left second finger when she was 53 years-old. The patient has had several superficial thrombophlebitis attacks since she was 34 years-old. She had no history of diabetes or hyperlipidemia, and had never smoked. On physical examination, her right foot and left second finger were amputated and the left foot felt cold and pale, the motilities of the left foot toes

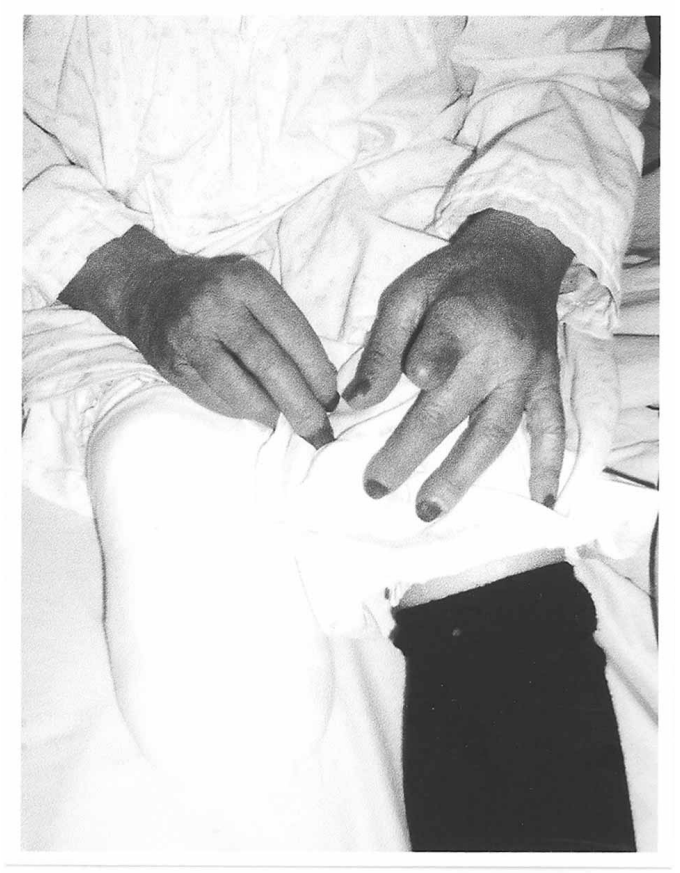

Figure 1. Photograph showing major and minor extremity amputations. 
were conserved (Figure 1). Her bilateral popliteal and left foot pulses were weakly palpable. She was mildly hypertensive. Renal functions were intact. There were no deficiencies of Protein C, Protein S or antithrombin III. Plasminogen, homocysteine, Leiden's factor $\mathrm{V}$, and cryoglobulin were within reference ranges. We recorded only fibrinogenemia of $510 \mathrm{mg} / \mathrm{dL}$ and an erythrocyte sedimentation rate (ESR) of $45 \mathrm{~mm}$ per hour. Echocardiography did not show any embolic cardiac sources.

There were diagnostic criteria of TAO in this patient. Rheumatoid factor (RF), anticentromere antibody, Scl 70, antineutrophil cytoplasmic autoantibody (ANCA) and AMA were negative. Among the nonorgan specific antibodies, only antinuclear antibody (ANA) was found in her serum. Lupus anticoagulants were negative; however, IgM anticardiolipin antibodies were detected. Anticardiolipin antibodies (aCLa) were again positive in repeated tests.

Arteriography showed regular patency of the aortic, iliac, femoral, and popliteal arteries with no visualization of the right tibial artery and occlusion of the left distal posterior tibial and peroneal arteries. The anterior tibial artery was filiform until its third medio-distal segment. Upper extremity arteriography showed bilaterally intact subclavian, brachial, radial, and ulnar artery systems. Left digital artery systems were patent except for the second finger. The ulnar artery was dominant on the right and obstructed immediately before the palmar

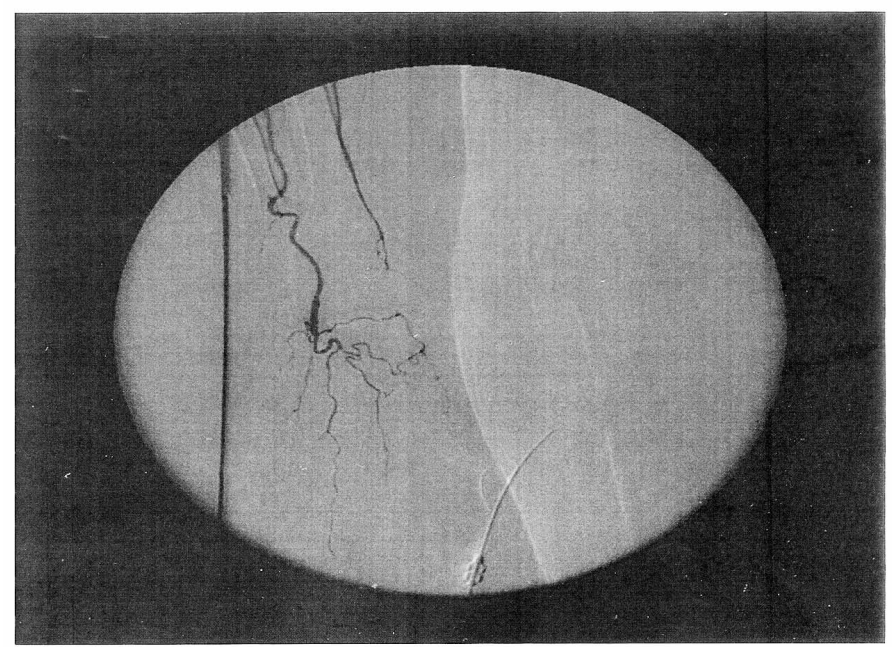

Figure 2. Angiogram showing occluded right ulnar artery immediately before palmar arch. 
arch (Figure-2). First and second digital arteries were visualized filiformly. Congestion was visualized at the third finger pulp.

During her 2 week hospitalization, she complained of a continuous resting finger pain with functional incompetence. Therapy consisting of ilioprost, antiplatelet administration, and heparinization did not provide any relief from the finger pain. After drug therapy proved ineffective, cervical sympathectomy was performed to relieve the finger pain. She described slight pain relief, but her finger ulcer did not show any signs of healing. Amputation of her dry digital gangrene was deferred until the lesion was fully demarcated.

\section{CASe 2}

A 34-year-old woman was observed for the first time for pain, paleness, hypothermia in her feet, and ulceration and dry gangrene of the right third toe (Figure 3). The patient described three superficial thrombophlebitis attacks in the last year. There was also a history of Raynaud's phenomenon. She had no history of miscarriage or abortions. She was severely hypertensive and nondiabetic. She had never smoked. Complete blood count was normal. Liver and renal function tests and lipid profile were all normal. Protein C, S, antithrombin III, plasminogen, homocysteine, Leiden's factor V, and cryoglobulin were within reference ranges. A high ESR of $65 \mathrm{~mm} /$ hour and hyperfibrinogenemia $(583 \mathrm{mg} / \mathrm{dL})$ were

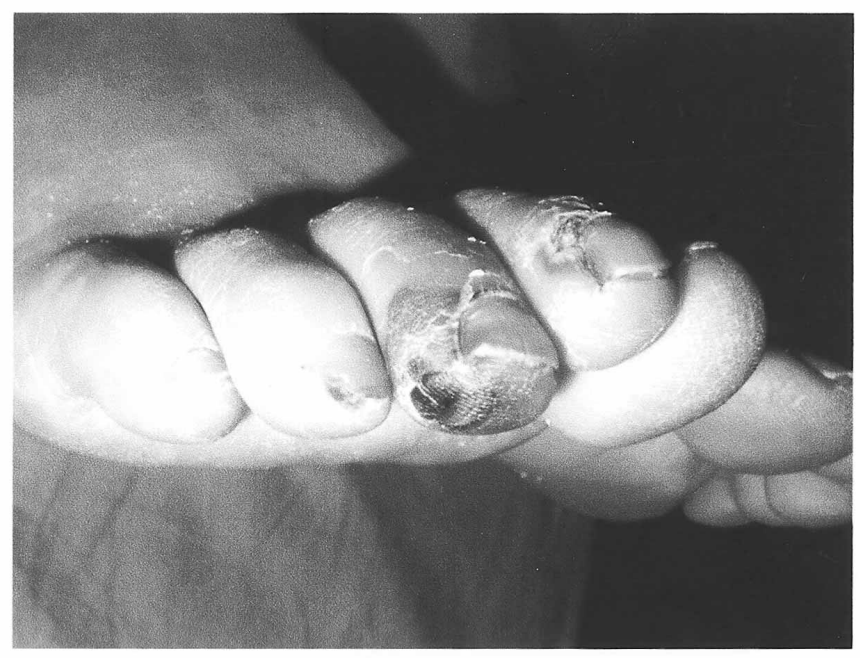

Figure 3. Photograph showing toe ulcer and dry gangrene. 


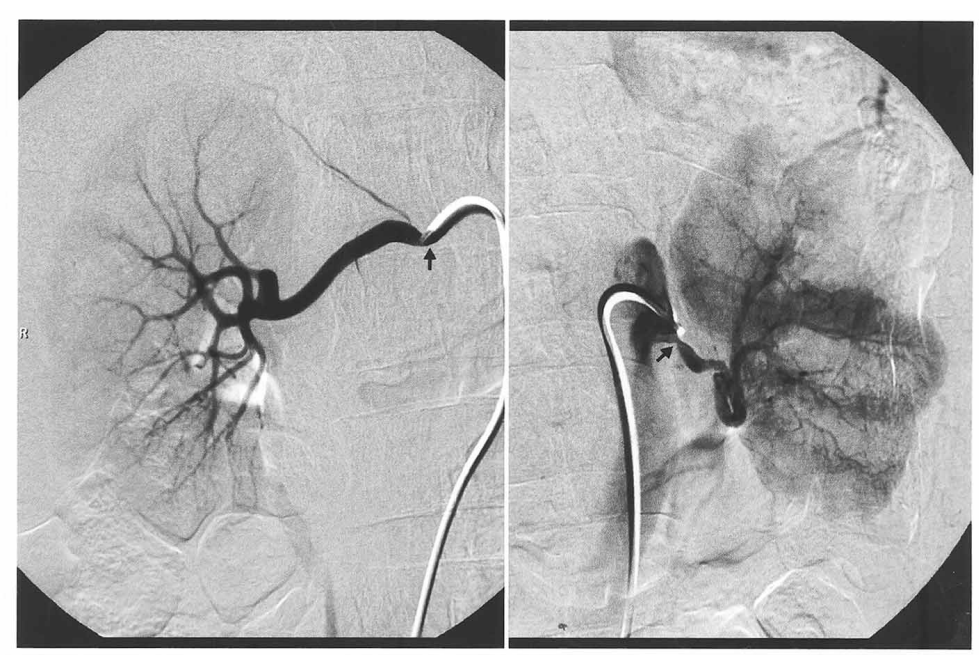

Figure 4. Angiogram showing bilateral renal artery orifice stenosis and thin and undulated left renal artery.

noted. RF, ANCA, ANA, anticentromere antibody, and Scl 70 were negative. Lupus anticoagulants were negative but $\operatorname{IgM}$ and $\mathrm{IgG}$ anticardiolipin antibodies were detected in the first and repeated serologic tests.

Echocardiography did not show any embolic cardiac sources. Echocardiography showed signs of left ventricular wall hypertrophy.

The patient had malignant hypertension. Although an intensive antihypertensive drug therapy regimen was adopted, her blood pressure decreased only to 160/110 mmHg. Selective renal arteriography showed hemodynamically important stenosis of bilateral renal artery origins (Figure 4). The left renal artery was thin and undulated and upper pole parenchymal dying was defective. Right renal cortical and parenchymal dying was normal.

Arteriography showed regular patency of the iliac, femoral, and popliteal arteries. The tibial arterial systems were bilaterally filiform until their third distal segments.

Stenting of the right renal artery mildly decreased blood pressure. Infusion therapy with prostanoid as well as antiplatelet and anticoagulant agents alleviated the right third toe pain. We did not consider amputation for the toe ulceration.

\section{DISCUSSION}

Buerger's disease or TAO is quite uncommon in Western countries, but is more prevalent in Eastern Europe, the Mediterranean Basin, Southeastern Asia, India, and the Far East. ${ }^{5)}$ 
Historically it has been described in males, young adults, and heavy smokers, however, recently it is affecting women more and more, probably because of the increased incidence of smoking among women. ${ }^{6}$ ) Diagnosis relies on angiography, with stenotic and obstructive lesions that often give the vessels a hypoplastic and filiform look (so-called mouse's tail) with collateral circulation around the obstructions, like a corkscrew. ${ }^{7)}$

Buerger's disease occurs in young adults, the median age at onset reported in the literature is 34 years. The reported male:female sex ratio is $7.5: 1,{ }^{8)}$ while only 2/500 patients described by Leo Buerger were women. ${ }^{9}{ }^{9}$ There has been a relative increase in the incidence of the disease in women reported in Europe and North America.

Kobayashi and colleagues suggested that Buerger's disease is an immunogenic condition likely initiated by an antigen in the intimal layer. Preservation of the internal elastic laminae distinguishes it from the true necrotizing forms of arteritis. The antigen responsible is still unknown. ${ }^{10)}$ The association of Buerger's disease and tobacco exposure appears undeniable, although the precise mechanism and possible inciting antigen(s) have yet to be characterized.

Sasaki, et al showed that the clinical features in female patients with TAO were equal to those in male patients, except for the fact that nonsmokers were more common in female patients. ${ }^{3)}$ Tobacco is strongly associated with the development and course of Buerger's disease, but the relative rarity of Buerger's disease despite widespread tobacco consumption is unexplained. The peculiar geographic and ethnic distribution of the disease suggests the importance of as yet unidentified environmental or genetic factors. We have described Buerger's disease in two nonsmoker females and speculate that the relation between tobacco consumption and Buerger's disease is not concrete.

Maslowski and colleagues showed that the prevalence of aCLa was significantly higher in patients with TAO (36\%) as compared to premature atherosclerosis $(8 \%)$ or healthy individuals $(2 \%)$. In their series, patients with TAO and a high antibody titer tended to be younger and suffer a significantly higher rate of major amputations compared to those without antibody. They suggested screening for aCLa in patients meeting the diagnostic criteria for TAO. ${ }^{11}$

Thromboangiitis obliterans (TAO) and antiphospholipid syndrome (AP) share the clinical characteristics of arterial thrombosis and recurrent thrombophlebitis. The majority of the thrombotic events in patients with antiphospholipid syndrome affect mainly the deep venous system of the lower limbs and arterial thrombosis predominates in the cerebral territory. ${ }^{12)}$ Patients have clinical features characterized by thrombocytopenia, recurrent abortion, and strokes, which were not present clinically and historically in both of our cases. 
Recently, it has been suggested that ANCA can be detected in patients with idiopathic necrotizing and crescentic glomerulonephritis or necrotizing angiitis, and can be used as a serological marker for ANCA-related systemic angiitis. ${ }^{13)}$ However, neither of our cases had ANCA-related systemic angiitis, as proven by the negative serology for ANCA and absence of signs of glomerulonephritis.

Although the association of anticardiolipin antibodies and TAO has been previously recognized, we showed anticardiolipin autoantibodies in two nonsmoker female TAO cases with poor prognoses. We suggest that screening for aCLa should be considered in patients meeting the diagnostic criteria for TAO.

\section{REFERENCES}

1. Nunez GA, Lopez CL, Rico ZL, Molins OA, Gomez OJ, Arribas A. Thromboangiitis obliterans (Buerger's disease) with intestinal involvement - a case report. Angiology 1998; 49: 489-92.

2. Swigris JJ, Olin JW, Mekhail NA. Implantable spinal cord stimulator to treat the ischemic manifestations of thromboangiitis obliterans (Buerger's disease). J Vasc Surg 1999; 29: 928-35.

3. Sasaki S, Sakuma M, Kunihara T, Yasuda K. Current trends in thromboangiitis obliterans (Buerger's disease) in women. Am J Surg 1999; 177: 316-20.

4. Papa M, Bass A, Adar R, et al. Autoimmune mechanisms in thromboangiitis obliterans (Buerger's disease): the role of tobacco antigen and the major histocompatibility complex. Surgery 1992; 11: 527-31.

5. Mills JL. Buerger's disease. Current Status. Vasc Med Rev 1994; 5: 139-50.

6. Olin JW, Young JR, Graor RA, et al. The changing clinical spectrum of thromboangiitis obliterans (Buerger's disease). Circulation 1990; 82 (5 Suppl): IV3-8.

7. Nielubowicz J, Rosnowski A, Pruszynski B, Przetakiewicz Z, Potemkowski A. Natural history of Buerger's disease. J Cardiovasc Surg (Torino) 1980; 21: 529-40.

8. Mills JL, Porter JM. Buerger's disease (thromboangiitis obliterans). Ann Vasc Surg 1991; 5: 570-2. (Review)

9. Buerger L. The Circulatory Disturbances of the Extremities Including Gangrene, Vasomotor and Trophic Disorders. Philadelphia, W.B. Saunders, Company, 1924.

10. Kobayashi M, Ito M, Nakagawa A, Nishikimi N, Nimura Y. Immunohistochemical analysis of arterial wall cellular infiltration in Buerger's disease (endarteritis obliterans). J Vasc Surg 1999; 29: 451-8.

11. Maslowski L, McBane R, Alexewicz P, Wysokinski WE. Antiphospholipid antibodies in thromboangiitis obliterans. Vasc Med 2002; 7: 259-64.

12. Merrill JT. Diagnosis of the antiphospholipid syndrome: how far to go? Curr Rheumatol Rep 2004; 6: 469-72.

13. Segelmark M, Westman K, Wieslander J. How and why should we detect ANCA? Clin Exp Rheumatol 2000; 18: 629-35. 